PROCEEDINGS OF THE

AMERICAN MATHEMATICAL SOCIETY

Volume 136, Number 10, October 2008, Pages 3467-3476

S 0002-9939(08)08998-3

Article electronically published on May 19, 2008

\title{
ON THE UNIQUENESS OF THE BROWDER DEGREE
}

\author{
J. BERKOVITS AND M. MIETTUNEN \\ (Communicated by Jonathan M. Borwein)
}

In memory of Juha Berkovits, who passed away on 3 August 2007

\begin{abstract}
We consider the topological degree theory for maximal monotone perturbations of mappings of class $\left(S_{+}\right)$originally introduced by F. Browder in 1983. In the original construction it is implicitly assumed that the maximal monotone part is at least densely defined. The construction itself remains valid without this assumption. However, for the proof of the uniqueness of the degree the assumption is crucial. We shall recall the construction of the degree and show how the stabilization of the degree can be obtained directly, thus avoiding a series of technical lemmas used by F. Browder. The main result of this paper is the proof for the uniqueness of the degree in the general case. We also discuss the class of admissible homotopies, which may be quite narrow in case the domain of the maximal monotone part is not densely defined.
\end{abstract}

\section{INTRODUCTION}

Let $X$ be a real reflexive Banach space and $T: X \rightarrow 2^{X^{*}}$ a given multivalued maximal monotone map. In [4] F. Browder, among other constructions, defined degree theory for mappings of the form $T+F$, where $F$ is demicontinuous bounded and of class $\left(S_{+}\right)$. It is a standard notation for multivalued mappings to write $T: X \rightarrow 2^{X^{*}}$, where the image $T(u)$ can be the empty set. In this note we prefer the notation

$$
T: D(T) \subset X \rightarrow 2^{X^{*}} \backslash \emptyset,
$$

where the domain of $T$ (often called effective domain) is

$$
D(T)=\{u \in X \mid T(u) \neq \emptyset\} .
$$

In 4 there is no explicitly stated assumption about the domain $D(T)$, and due to the notation $T: X \rightarrow 2^{X^{*}}$ it is not clear at first sight whether or not some is actually needed. However, by a closer eximination of the proofs one can see that it is implicitly assumed that $D(T)$ is at least dense in $X$. The main point is that the use of affine homotopies is not possible without $D(T)$ being dense, thus destroying the proof of the uniqueness of the degree. We also want to point out that in the paper of S. Hu and N. Papageorgiou [6] the uniqueness proof of a further extension of the degree should be modified accordingly, since their construction is based on the original paper of Browder without any assumption on $D(T)$. The

Received by the editors June 5, 2006.

2000 Mathematics Subject Classification. Primary 47H11, 47H05. $\left(S_{+}\right)$.

Key words and phrases. Uniqueness of the degree, maximal monotonicity, mappings of class 
missing assumptions in Browder's construction is also noted in the recent article by M. Otani and J. Kobayashi 7. The main emphasis of Otani and Kobayashi lies on the applications to variational inequalities. Our main result is the uniqueness of the degree.

The paper is organized as follows. In Section 2 we recall some definitions and basic properties of the mappings needed. Section 3 is devoted to the construction of the degree. We briefly indicate how the original proof of F. Browder can be simplified and avoid some technical lemmas. In Sections 45 we discuss the properties of the degree. The main emphasis is on the class of admissible homotopies. It turns out that generally the affine homotopies cannot be used. This will severely restrict the practical use of the degree. In view of applications it is important to have mappings for which the degree is nonzero - a few such are given at the end of Section 4 In Section 6 we shall prove our main result that the degree function is unique for any domain $D(T)$ of the maximal monotone part.

\section{Mappings of monotone type}

We start with some well-known facts about maximal monotone mappings and other classes of monotone type. Let $X$ be a real reflexive Banach space. Note that by the results of Troyanski and Kadec (see [5], for instance) we can assume that both $X$ and $X^{*}$ are locally uniformly convex. Let $T: D(T) \subset X \rightarrow 2^{X^{*}} \backslash \emptyset$ be a given multivalued mapping. We say that $T$ is monotone, if

$$
\langle w-y, u-x\rangle \geq 0 \text { for all } u, x \in D(T), w \in T(u), y \in T(x) .
$$

The multivalued map $T$ is maximal monotone, if it is monotone and the graph

$$
\mathcal{G}(T)=\{(u, w) \mid u \in D(T), w \in T(u)\}
$$

is maximal monotone set in $X \times X^{*}$, that is, $T$ is monotone and if for some pair $(x, y) \in X \times X^{*}$

$$
\langle w-y, u-x\rangle \geq 0 \text { for all }(u, w) \in \mathcal{G}(T),
$$

then $(x, y) \in \mathcal{G}(T)$. It is easy to see that $T: D(T) \subset X \rightarrow 2^{X^{*}} \backslash \emptyset$ is maximal monotone if and only if $T^{-1}: R(T) \rightarrow 2^{X} \backslash \emptyset$ is maximal monotone.

For our later use we recall some definitions of mappings of monotone type. A single valued mapping $F: X \rightarrow X^{*}$ is quasimonotone, if $u_{k} \rightarrow u$ (weak convergence) in $X$ implies $\lim \sup \left\langle F\left(u_{k}\right), u_{k}-u\right\rangle \geq 0$. Mapping $F$ is pseudomonotone, if $u_{k} \rightarrow u$ in $X$ and $\lim \sup \left\langle F\left(u_{k}\right), u_{k}-u\right\rangle \leq 0$ imply $F\left(u_{k}\right) \rightarrow F(u)$ and $\left\langle F\left(u_{k}\right), u_{k}-u\right\rangle \rightarrow 0$. $\mathrm{F}$ is of class $\left(S_{+}\right)$, if $u_{k} \rightarrow u$ in $X$ and $\lim \sup \left\langle F\left(u_{k}\right), u_{k}-u\right\rangle \leq 0$ imply $u_{k} \rightarrow u$. For mappings of monotone type and the mutual relations between different classes we refer to [1] and 4, for instance. If all the mappings are demicontinuous and bounded in the sense that the image of any bounded set remains bounded, then we have with obvious notations the inclusions

$$
\left(S_{+}\right) \subset(P M) \subset(Q M) .
$$

Moreover, for demicontinuous and bounded maps the following hold: any monotone map is pseudomonotone and any compact map is quasimonotone. The class $\left(S_{+}\right)$ is stable under quasimonotone perturbations. Homotopies of monotone type will be defined in Sections 3 and 4 .

We recall that the duality map $J: X \rightarrow X^{*}$ is the unique mapping defined by properties $\langle J(u), u\rangle=\|u\|^{2}$ and $\|J(u)\|=\|u\|$ for all $u \in X$. If $X$ and $X^{*}$ are locally uniformly convex, then $J$ is single valued, bijective, bicontinuous, maximal 
monotone, strictly monotone, bounded and of class $\left(S_{+}\right)$. In addition $J^{-1}: X^{*} \rightarrow$ $X$ is the duality map of the dual space $X^{*}$, where we have identified $X$ with $X^{* *}$ by reflexivity. In fact the duality map satisfies a stronger condition than $\left(S_{+}\right)$. Indeed, for any sequence $\left(u_{k}\right) \subset X$ such that for some $u \in X$

$$
\lim \left\langle J\left(u_{k}\right)-J(u), u_{k}-u\right\rangle=0,
$$

it follows that $u_{k} \rightarrow u$ (see [8, p.864). The following lemma is proved in [8, p. 886 (see also [3]), for instance. Both $X$ and $X^{*}$ are assumed to be locally uniformly convex.

Lemma 2.1. Let $T: D(T) \subset X \rightarrow 2^{X^{*}} \backslash \emptyset$ be monotone. Then $T$ is maximal monotone if and only if $T+\lambda J$ is surjective for all $\lambda>0$.

It is easy to see that any maximal monotone map satisfies the following generalized pseudomonotonicity condition.

Lemma 2.2. Assume that $T: D(T) \subset X \rightarrow 2^{X^{*}} \backslash \emptyset$ is maximal monotone. Then $T$ satisfies the generalized pseudomonotonicity condition: for any sequences $\left(u_{k}\right) \subset$ $D(T)$ and $\left(w_{k}\right), w_{k} \in T\left(u_{k}\right)$ such that

$$
u_{k} \rightarrow u \text { in } X, w_{k} \rightarrow w \text { in } X^{*} \text { and } \lim \sup \left\langle w_{k}, u_{k}-u\right\rangle \leq 0,
$$

it follows that $u \in D(T), w \in T(u)$ and $\lim \left\langle w_{k}, u_{k}-u\right\rangle=0$.

\section{Construction of Degree}

Let $X$ be a real reflexive Banach space such that $X$ and $X^{*}$ are locally uniformly convex. For any maximal monotone map $T: D(T) \rightarrow 2^{X^{*}} \backslash \emptyset$ we associate the family of Yosida transformations

$$
T_{\varepsilon}=\left(T^{-1}+\varepsilon J^{-1}\right)^{-1}, \quad \varepsilon>0 .
$$

By Lemma 2.1 it is clear that $D\left(T_{\varepsilon}\right)=X$. It is not hard to see that $T_{\varepsilon}: X \rightarrow X^{*}$ is single valued. For the proof of the following lemma, see [4].

Lemma 3.1. Let $T: D(T) \rightarrow 2^{X^{*}} \backslash \emptyset$ be a maximal monotone map with $0 \in T(0)$ and let $F: \bar{G} \rightarrow X^{*}$ be a bounded demicontinuous map of class $\left(S_{+}\right)$, where $G \subset X$ is an open bounded set.

a) Then $T_{\varepsilon}=\left(T^{-1}+\varepsilon J^{-1}\right)^{-1}: X \rightarrow X^{*}$ is bounded, continuous, maximal monotone and pseudomonotone for all $\varepsilon>0$.

b) Assume that $y_{0} \notin(T+F)(A \cap D(T))$, where $A \subset \bar{G}$ is closed. Then there exists $\varepsilon_{0}>0$ such that $y_{0} \notin\left(T_{\varepsilon}+F\right)(A)$ for all $0<\varepsilon<\varepsilon_{0}$.

The following two results are needed in order to simplify the original construction of F.Browder. Let $T: D(T) \rightarrow 2^{X^{*}} \backslash \emptyset$ be a maximal monotone map. Without loss of generality we assume $0 \in T(0)$. The first lemma is a continuity property.

Lemma 3.2. Assume that $u_{k} \rightarrow u$ in $X$ and $\varepsilon_{k} \rightarrow \varepsilon>0$. Then $T_{\varepsilon_{k}}\left(u_{k}\right) \rightarrow T_{\varepsilon}(u)$ in $X^{*}$.

Proof. Denote $w_{k}=T_{\varepsilon_{k}}\left(u_{k}\right)$. Then $u_{k}=v_{k}+\varepsilon_{k} J^{-1}\left(w_{k}\right)$ with some $v_{k} \in T^{-1}\left(w_{k}\right)$. By the monotonicity of $T^{-1}$ we get

$$
\left\|w_{k}\right\|\left\|u_{k}\right\| \geq\left\langle w_{k}, u_{k}\right\rangle=\left\langle w_{k}, v_{k}\right\rangle+\varepsilon_{k}\left\langle w_{k}, J^{-1}\left(w_{k}\right)\right\rangle \geq \varepsilon_{k}\left\|w_{k}\right\|^{2}
$$

and consequently $\left(w_{k}\right)$ is bounded. It is easy to see that

$$
T_{\varepsilon_{k}}\left(u_{k}\right) \equiv T_{\varepsilon}\left(u_{k}+\left(\varepsilon-\varepsilon_{k}\right) J^{-1}\left(w_{k}\right)\right) \text {. }
$$


Hence the conclusion follows by the continuity of $T_{\varepsilon}$.

The second lemma can be wieved as a pseudomonotonicity type property.

Lemma 3.3. Assume that $u_{k} \rightarrow u$ in $X, \varepsilon_{k} \rightarrow \varepsilon>0$ and $\lim \sup \left\langle T_{\varepsilon_{k}}\left(u_{k}\right), u_{k}-u\right\rangle \leq$ 0 . Then $T_{\varepsilon_{k}}\left(u_{k}\right) \rightarrow T_{\varepsilon}(u)$ and $\left\langle T_{\varepsilon_{k}}\left(u_{k}\right), u_{k}-u\right\rangle \rightarrow 0$.

Proof. Denote $w_{k}=T_{\varepsilon_{k}}\left(u_{k}\right)$. Like in proof of Lemma 3.2 we see that $\left(w_{k}\right)$ is bounded. The rest of the proof follows easily from the pseudomonotonicity of $T_{\varepsilon}$, since

$$
w_{k}=T_{\varepsilon}\left(u_{k}+\left(\varepsilon-\varepsilon_{k}\right) J^{-1}\left(w_{k}\right)\right) .
$$

As a direct consequence of the above results we get the next lemma.

Lemma 3.4. Let $T: D(T) \rightarrow 2^{X^{*}} \backslash \emptyset$ be a maximal monotone map with $0 \in T(0)$ and let $F: \bar{G} \rightarrow X^{*}$ be a bounded demicontinuous map of class $\left(S_{+}\right)$, where $G \subset X$ is an open bounded set. Let $0<\underline{\varepsilon}<\bar{\varepsilon}$ and denote

$$
H(\varepsilon, u)=T_{\varepsilon}(u)+F(u), \quad u \in \bar{G} \text { and } \underline{\varepsilon} \leq \varepsilon \leq \bar{\varepsilon} .
$$

Then $H(\cdot, \cdot)$ is a bounded homotopy of class $\left(S_{+}\right)$, i.e., $H(\cdot, \cdot)$ is bounded demicontinuous and for any sequences $\left(\varepsilon_{k}\right) \subset[\varepsilon, \bar{\varepsilon}], \varepsilon_{k} \rightarrow \varepsilon,\left(u_{k}\right) \subset X, u_{k} \rightarrow u$, such that $\lim \sup \left\langle H\left(\varepsilon_{k}, u_{k}\right), u_{k}-u\right\rangle \leq 0$, it follows that $u_{k} \rightarrow u$ and $H\left(\varepsilon_{k}, u_{k}\right) \rightarrow H(\varepsilon, u)$.

Hence the stabilization of the degree can be easily proved.

Theorem 3.5. With the assumptions of Lemma 3.4 let $y_{0} \notin(T+F)(\partial G \cap D(T))$. Then there exists $\varepsilon_{0}>0$ such that $y_{0} \notin\left(T_{\varepsilon}+F\right)(\partial G)$ and

$$
d_{S_{+}}\left(T_{\varepsilon}+F, G, y_{0}\right)=\text { constant for all } 0<\varepsilon<\varepsilon_{0} .
$$

Proof. Applying Lemma $3.1 \mathrm{~b})$ with $A=\partial G$ we obtain $\varepsilon_{0}>0$ such that $y_{0} \notin$ $\left(T_{\varepsilon}+F\right)(\partial G)$ for all $0<\varepsilon<\varepsilon_{0}$. Let $0<\varepsilon_{1}<\varepsilon_{2}<\varepsilon_{0}$ be fixed. By Lemma 3.4 $H(\varepsilon, u)=T_{\varepsilon}(u)+F(u), u \in \bar{G}, \varepsilon_{1} \leq \varepsilon \leq \varepsilon_{2}$, defines a bounded homotopy of class $\left(S_{+}\right)$. Consequently, $\mathrm{d}_{S_{+}}\left(H(\varepsilon, \cdot), G, y_{0}\right)$ remains constant for all $\varepsilon_{1} \leq \varepsilon \leq \varepsilon_{2}$. Hence $\mathrm{d}_{S_{+}}\left(T_{\varepsilon_{1}}+F, G, y_{0}\right)=\mathrm{d}_{S_{+}}\left(T_{\varepsilon_{2}}+F, G, y_{0}\right)$, completing the proof.

Denote by $\mathrm{d}_{S_{+}}$the unique degree function for demicontinuous mappings of class $\left(S_{+}\right)$(see [4]). Define

$$
\mathrm{d}\left(T+F, G, y_{0}\right)=\lim _{\varepsilon \rightarrow 0+} \mathrm{d}_{S_{+}}\left(T_{\varepsilon}+F, G, y_{0}\right)
$$

for any maximal monotone $T: D(T) \rightarrow 2^{X^{*}} \backslash \emptyset$ with $0 \in T(0)$, and for any bounded demicontinuous map $F: \bar{G} \rightarrow X^{*}$ which is of class $\left(S_{+}\right)$, where $G$ is an open bounded set in $X$ and $y_{0} \notin(T+F)(\partial G \cap D(T))$.

\section{Properties of Degree}

The integer valued function d defined by (3.1) is a degree function, provided the next four conditions hold. The class of admissible homotopies referred to in (c) will be defined later in this section. Let $y_{0} \notin(T+F)(\partial G \cap D(T))$.

(a) If $\mathrm{d}\left(T+F, G, y_{0}\right) \neq 0$, then there exists $u \in G \cap D(T)$ such that $y_{0} \in$ $(T+F)(u)$.

(b) Let $G_{1}, G_{2} \subset G$ and $G_{1} \cap G_{2}=\emptyset$. If $y_{0} \notin(T+F)\left(\left(\bar{G} \backslash\left(G_{1} \cup G_{2}\right)\right) \cap D(T)\right)$, then $\mathrm{d}\left(T+F, G, y_{0}\right)=\mathrm{d}\left(T+F, G_{1}, y_{0}\right)+\mathrm{d}\left(T+F, G_{2}, y_{0}\right)$. 
(c) Let $\left\{H_{t} \mid t \in[0,1]\right\}$ be a admissible homotopy and $\{y(t) \mid t \in[0,1]\}$ a continuous curve such that $y(t) \notin H_{t}(\partial G)$ for all $t \in[0,1]$. Then $\mathrm{d}\left(H_{t}, G, y(t)\right)$ is constant for all $t \in[0,1]$.

(d) Normalization: $\mathrm{d}\left(J, G, y_{0}\right)=+1$ for all $y_{0} \in J(G)$.

The basic properties (a), (b) and (d) are easily verified. As usual, the homotopy invariance property $(\mathrm{c})$ requires some extra work.

We start with the definition inspired by Lemma 2.2. Let $\left\{T_{t} \mid 0 \leq t \leq 1\right\}$ be a family of multis. We say that $T_{t}$ is a pseudomonotone homotopy of maximal monotone mappings, denoted $T_{t} \in(P M)_{M M}$, if

(i) $0 \in T_{t}(0)$ and $T_{t}: D\left(T_{t}\right) \subset X \rightarrow 2^{X^{*}} \backslash \emptyset$ is maximal monotone for all $t \in[0,1]$,

(ii) for any sequences $\left(t_{k}\right) \subset[0,1],\left(u_{k}\right), u_{k} \in D\left(T_{t_{k}}\right)$, and $\left(w_{k}\right), w_{k} \in T_{t_{k}}\left(u_{k}\right)$, such that

$$
u_{k} \rightarrow u \text { in } X, t_{k} \rightarrow t, w_{k} \rightarrow w \text { in } X^{*} \text { and } \lim \sup \left\langle w_{k}, u_{k}-u\right\rangle \leq 0,
$$

it follows that $u \in D\left(T_{t}\right), w \in T_{t}(u)$ and $\lim \left\langle w_{k}, u_{k}-u\right\rangle=0$.

Useful characterizations of a pseudomonotone homotopy of maximal monotone maps are given in the next result (see [4]).

Lemma 4.1. Let $\left\{T_{t} \mid 0 \leq t \leq 1\right\}$ be a family of maximal monotone multis such that $0 \in T_{t}(0)$ for each $t \in[0,1]$. Then condition (ii) above and the following three conditions are mutually equivalent:

(1) The map defined by $\psi(t, w)=\left(T_{t}+J\right)^{-1}(w)$ is continuous from $[0,1] \times X^{*}$ into $X$.

(2) For each fixed $w \in X^{*}$ the map defined by $\psi_{w}(t)=\left(T_{t}+J\right)^{-1}(w)$ is continuous from $[0,1]$ into $X$.

(3) For any given pair $(u, w), w \in T_{t}(u)$, and any sequence $\left(t_{k}\right), t_{k} \rightarrow t$, there exist sequences $\left(u_{k}\right)$ and $\left(w_{k}\right)$ such that $w_{k} \in T_{t_{k}}\left(u_{k}\right)$ and $u_{k} \rightarrow u, w_{k} \rightarrow w$.

As in Lemma 3.1 a) we have for homotopies the following result.

Lemma 4.2. Assume $T_{t} \in(P M)_{M M}$. Then, for any fixed $\varepsilon>0$ the family $\left\{\left(T_{t}\right)_{\varepsilon} \mid 0 \leq t \leq 1\right\}$ is a bounded pseudomonotone homotopy, i.e., for any sequences $\left(t_{k}\right) \subset[0,1],\left(u_{k}\right) \subset X$, such that

$$
u_{k} \rightarrow u \text { in } X, t_{k} \rightarrow t \text { and } \lim \sup \left\langle\left(T_{t_{k}}\right)_{\varepsilon}\left(u_{k}\right), u_{k}-u\right\rangle \leq 0,
$$

it follows that $\lim \left\langle\left(T_{t_{k}}\right)_{\varepsilon}\left(u_{k}\right), u_{k}-u\right\rangle=0$ and $\left(T_{t_{k}}\right)_{\varepsilon}\left(u_{k}\right) \rightarrow\left(T_{t}\right)_{\varepsilon}(u)$.

We shall need only the fact that $\left(T_{t}\right)_{\varepsilon}$ is quasimonotone and bounded (demi)continuous map from $[0,1] \times X$ to $X^{*}$. The quasimonotonicity condition

$$
u_{k} \rightarrow u \text { in } X \text { and } t_{k} \rightarrow t \text { in }[0,1] \text { imply } \lim \sup \left\langle\left(T_{t_{k}}\right)_{\varepsilon}\left(u_{k}\right), u_{k}-u\right\rangle \geq 0
$$

is clearly satisfied by Lemma 4.2 .

Let $\left\{F_{t} \mid 0 \leq t \leq 1\right\}$ be a family of mappings from $\bar{G}$ to $X^{*}$, where $G \subset X$ is an open bounded set. We say that $F_{t}$ is a homotopy of class $\left(S_{+}\right)$if for any sequences $\left(t_{k}\right) \subset[0,1]$ and $\left(u_{k}\right) \subset \bar{G}$ such that

$$
u_{k} \rightarrow u \text { in } X, \quad t_{k} \rightarrow t \text { and } \lim \sup \left\langle F_{t_{k}}\left(u_{k}\right), u_{k}-u\right\rangle \leq 0,
$$

it follows that $u_{k} \rightarrow u$ and $F_{t_{k}}\left(u_{k}\right) \rightarrow F_{t}(u)$. The admissible homotopies are of the form $T_{t}+F_{t}, 0 \leq t \leq 1$, where $T_{t} \in(P M)_{M M}$ and $F_{t}$ is a bounded homotopy of the class $\left(S_{+}\right)$. In view of Lemma 4.2 it is clear that $\left(T_{t}\right)_{\varepsilon}+F_{t}$ defines a bounded 
homotopy of the class $\left(S_{+}\right)$for each $\varepsilon>0$. The homotopy invariance property follows from the following result.

Lemma 4.3. Assume that $y(t) \notin\left(T_{t}+F_{t}\right)\left(\partial G \cap D\left(T_{t}\right)\right)$ for all $0 \leq t \leq 1$. Then there exists $\varepsilon_{0}>0$ such that $y(t) \notin\left(\left(T_{t}\right)_{\varepsilon}+F_{t}\right)(\partial G)$ for all $0 \leq t \leq 1$ and $0<\varepsilon<\varepsilon_{0}$. Moreover,

$$
d_{S_{+}}\left(\left(T_{t}\right)_{\varepsilon}+F_{t}, G, y(t)\right)=\text { constant for all } 0 \leq t \leq 1 \text { and } 0<\varepsilon<\varepsilon_{0} .
$$

In order to use the homotopy invariance property of degree effectively it is important to have "reference mappings", that is, mappings with nonzero degree. The duality map $J$ which serves as a normalizing map is not always the best alternative. We have the following simple observation.

Theorem 4.4. Let $T$ be any maximal monotone mappings such that $0 \in T(0)$. Let $G \subset X$ be an open bounded set and assume that $w \in(T+J)(G \cap D(T))$. Then

$$
d(T+J, G, w)=+1 .
$$

Proof. The solution $u_{0} \in G$ of $w \in(T+J)(u)$ is unique and $\left\|u_{0}\right\| \leq\|w\|$. Thus $w \notin(T+J)(\partial G \cap D(T))$ and the degree is well-defined. Take any $R>0$ such that $R>\|w\|$. Then by the additivity property (b) of the degree

$$
\mathrm{d}(T+J, G, w)=\mathrm{d}\left(T+J, B_{R}(0), w\right) .
$$

It is easy to see that $t w \notin(T+J)\left(\partial B_{R}(0) \cap D(T)\right)$ for all $0 \leq t \leq 1$ and hence $\mathrm{d}\left(T+J, B_{R}(0), w\right)=\mathrm{d}\left(T+J, B_{R}(0), 0\right)$ by the homotopy invariance property. By definition $\mathrm{d}\left(T+J, B_{R}(0), 0\right)=\mathrm{d}_{S_{+}}\left(T_{\varepsilon}+J, B_{R}(0), 0\right)$ for all $\varepsilon>0$ small enough. By the monotonicity of $T_{\varepsilon}$ the equation $\left(t T_{\varepsilon}+J\right)(u)=0$ has only the trivial solution $u=0$ for all $0 \leq t \leq 1$. Consequently

$$
\mathrm{d}(T+J, G, w)=\mathrm{d}_{S_{+}}\left(T_{\varepsilon}+J, B_{R}(0), 0\right)=\mathrm{d}_{S_{+}}\left(J, B_{R}(0), 0\right)=+1,
$$

completing the proof.

As an example of the use of the previous lemma we have the following well-known existence result.

Corollary 4.5. Let $T$ be any maximal monotone mappings such that $0 \in T(0)$ and $D(T)$ is bounded. Let $F: X \rightarrow X^{*}$ be any bounded demicontinuous map of class $\left(S_{+}\right)$. Then the problem

$$
w \in(T+F)(u), \quad u \in D(T),
$$

admits at least one solution for any $w \in X^{*}$.

Proof. For any $R>0$ such that $D(T) \subset B_{R}(0)$ trivially

$$
w \notin(T+(1-t) F+t J)\left(\partial B_{R}(0) \cap D(T)\right)
$$

for all $0 \leq t \leq 1$. Hence the conclusion follows by Lemma 4.4.

The fact that the bounded domain $D(T)$ makes $T+F$ surjective reflects the maximality of $T$ which dominates the behavior of $T$ on the boundary of $D(T)$. If $D(T)$ is not bounded, some coercivity condition is needed. We close this section by a generalization of Borsuk's theorem of the degree of odd mappings. 
Theorem 4.6. Let $T$ be maximal monotone mappings such that $0 \in T(0)$ and $D(T)$ is symmetric with respect to the origin. Assume that $T$ is odd in the sense that if $w \in$ $T(-u)$, then $-w \in T(u)$. Let $F: X \rightarrow X^{*}$ be any bounded demicontinuous map of class $\left(S_{+}\right)$which is odd on $\partial B_{R}(0)$ for some $R>0$. If $0 \notin(T+F)\left(\partial B_{R}(0) \cap D(T)\right)$, then

$$
d\left(T+F, B_{R}(0), 0\right)=o d d .
$$

Proof. It is easy to see that $T_{\varepsilon}$ is odd. For some sufficiently small $\varepsilon>0$ we have

$$
\mathrm{d}\left(T+F, B_{R}(0), 0\right)=\mathrm{d}_{S_{+}}\left(T_{\varepsilon}+F, B_{R}(0), 0\right)=\text { odd },
$$

where the last equality follows from the corresponding result for mappings of class $\left(S_{+}\right)$(see [1]).

A standard example of maximal monotone mappings is the subdifferential $\partial \chi_{C}$ of the indicator function of a nonempty closed convex set $C$. It is not hard to see that $T=\partial \chi_{C}$ satisfies the assumtions of the previous theorem provided $0 \in C$ and $C$ is symmetric with respect to the origin. Hence Theorem 4.6 can naturally be applied to variational inequalities.

\section{INVARIANCE UNDER AFFINE HOMOTOPIES}

In view of applications of any degree theory the class of admissible homotopies is crucial. We call $F_{t}, 0 \leq t \leq 1$, an affine homotopy between $F_{0}$ and $F_{1}$, if it is of the form

$$
F_{t}=(1-t) F_{0}+t F_{1}, \quad 0 \leq t \leq 1 .
$$

Affine homotopies, being the simplest ones, are important for applications. Our admissible homotopies are of the form $T_{t}+F_{t}, 0 \leq t \leq 1$, where $T_{t} \in(P M)_{M M}$ is a pseudomonotone homotopy of maximal monotone mappings such that $0 \in T_{t}(0)$ for all $0 \leq t \leq 1$, and $F_{t}$ is a bounded homotopy of the class $\left(S_{+}\right)$. It is a well-known fact that the sum of two maximal monotone mappings is not necessarily maximal monotone (see [3], 8], p. 888). Hence, for arbitrary maximal monotone mappings $T$ and $S$ it is possible that $(1-t) T+t S$ is not even maximal monotone. Hence it is clear that in general not all affine homotopies are included to the class of pseudomonotone homotopies of maximal monotone mappings. There are two possible ways to define an affine homotopy. Indeed, let $T$ and $S$ be maximal monotone mappings such that $0 \in T(0)$ and $0 \in S(0)$. First, according to standard rules (see [8], p. 851)

$$
T_{t}=(1-t) T+t S, \quad D\left(T_{t}\right)=D(T) \cap D(S), \quad 0 \leq t \leq 1 .
$$

Secondly, we can define an affine homotopy piecewise by setting

$$
\hat{T}_{t}(u)=\left\{\begin{array}{l}
T(u), \quad t=0 \text { and } u \in D(T), \\
(1-t) T(u)+t S(u), \quad 0<t<1 \text { and } u \in D(T) \cap D(S), \\
S(u), \quad t=1 \text { and } u \in D(S), \\
\emptyset, \quad \text { otherwise. }
\end{array}\right.
$$

We shall examine both cases more closely.

Lemma 5.1. Let $T$ and $S$ be maximal monotone mappings such that $0 \in T(0)$ and $0 \in S(0)$. Homotopies $T_{t}, 0 \leq t \leq 1$, and $\hat{T}_{t}, 0 \leq t \leq 1$, are defined by (5.1) and (5.2), accordingly. Then

(i) $T_{t} \in(P M)_{M M}$ if and only if $D(T)=D(S)$. 
(ii) If $D(T)=D(S)$, then $\hat{T}_{t} \in(P M)_{M M}$.

(iii) If $\hat{T}_{t} \in(P M)_{M M}$, then $\overline{D(T)}=\overline{D(S)}=\overline{D(T) \cap D(S)}$.

Proof. (i) Assume first that $T_{t} \in(P M)_{M M}$. We need only the maximality to see that $D(T) \cap D(S)=D(T)$. Indeed, otherwise $D\left(T_{0}\right)$ would be a proper subset of $D(T)$ implying $T_{0}$ would be a restriction of $T$. But this contradicts the assumption that $T_{0}$ is maximal as a monotone map. Similarly $D(T) \cap D(S)=D(S)$ and thus $D(T)=D(S)$. We shall use condition (3) of Lemma 4.1 to prove our assertion. First, let $\left.t_{k} \rightarrow t \in\right] 0,1\left[\right.$ and $(u, w) \in \mathcal{G}\left(T_{t}\right)$. Because of $w \in(1-t) T(u)+t S(u)$ we can choose $x \in T(u)$ and $y \in S(u)$ such that $w=(1-t) x+t y$. We set $u_{k} \equiv u$ and $w_{k}=\left(1-t_{k}\right) x+t_{k} y$. Then $u_{k} \in D(T) \cap D(S)=D\left(\left(1-t_{k}\right) T+t_{k} S\right)$, $w_{k} \in\left(1-t_{k}\right) T\left(u_{k}\right)+t_{k} S\left(u_{k}\right)$ and $u_{k} \rightarrow u, w_{k} \rightarrow w$. Second, let $t_{k} \rightarrow 0$ and $(u, w) \in \mathcal{G}\left(T_{0}\right)=\mathcal{G}(T)$, that is, $u \in D(T)=D(S)$ and $w \in T(u)$. We set $u_{k} \equiv u$ and $w_{k}=\left(1-t_{k}\right) w+t_{k} x$, where $x \in S(u)$. It is easy to see that this leads to the desired result. Similarly condition (3) holds if $t=1$.

(ii) The second assertion is a trivial consequence of (i), since $T_{t}=\hat{T}_{t}$ in the case $D(T)=D(S)$.

(iii) Assume that $\hat{T}_{t} \in(P M)_{M M}$. Let $(u, w) \in \mathcal{G}\left(T_{t}\right)$ and $0<t_{k}<1, t_{k} \rightarrow 0$. By condition (3) of Lemma 4.1 there exist sequences $\left(u_{k}\right)$ and $\left(w_{k}\right)$ such that $\left(u_{k}, w_{k}\right) \in \mathcal{G}\left(T_{t_{k}}\right)$ and $u_{k} \rightarrow u, w_{k} \rightarrow w$. Because of $u_{k} \in D\left(T_{t_{k}}\right)=D(T) \cap D(S)$ and $u_{k} \rightarrow u$ we have $D(T) \subset \overline{D(T) \cap D(S)}$ and consequently $\overline{D(T)}=\overline{D(T) \cap D(S)}$. Similarly we get $\overline{D(S)}=\overline{D(T) \cap D(S)}$, completing the proof.

The homotopy of our next lemma is used in the original uniqueness proof of the degree given in [4]. Hence we will see that assumtion $\overline{D(T)}=X$ is necessary in [4].

Lemma 5.2. Let $T$ be maximal monotone mappings such that $0 \in T(0)$. Denote

$$
\begin{aligned}
& T_{t}(u)=(1-t) T(u), \quad u \in D\left(T_{t}\right)=D(T), \quad 0 \leq t \leq 1, \\
& \hat{T}_{t}(u)=\left\{\begin{array}{l}
(1-t) T(u), \quad 0 \leq t<1 \text { and } u \in D(T), \\
0, \quad t=1 \text { and } u \in X, \\
\emptyset, \quad \text { otherwise. }
\end{array}\right.
\end{aligned}
$$

Then

(i) $T_{t} \in(P M)_{M M}$ if and only if $D(T)=X$.

(ii) $\hat{T}_{t} \in(P M)_{M M}$ if and only if $\overline{D(T)}=X$.

Proof. Let $S=0$ with $D(S)=X$. Assertion (i) then follows from Lemma 5.1 (i). In view of Lemma 5.1 (ii) assertion (ii) is proved, as soon as we show that $\hat{T}_{t}$ is a pseudomonotone homotopy of maximal monotone mappings in case $D(T)$ is dense in $X$. Indeed, assume that $\overline{D(T)}=X$. Take any sequences $\left(t_{k}\right) \subset[0,1],\left(u_{k}\right)$, $u_{k} \in D\left(T_{t_{k}}\right)$, and $\left(w_{k}\right), w_{k} \in T_{t_{k}}\left(u_{k}\right)$ such that

$$
u_{k} \rightarrow u \text { in } X, t_{k} \rightarrow t, w_{k} \rightarrow w \text { in } X^{*} \text { and } \lim \sup \left\langle w_{k}, u_{k}-u\right\rangle \leq 0 .
$$

We shall show that $u \in D\left(T_{t}\right), w \in T_{t}(u)$ and $\lim \left\langle w_{k}, u_{k}-u\right\rangle=0$. If $t \neq 1$, there are no difficulties due to the fact that $T$ is maximal monotone and thus satisfies the generalized pseudomonotonicity condition (Lemma 2.2). Thus we assume that $t=1$, i.e., $t_{k} \rightarrow 1$. Without loss of generality we can assume that $t_{k} \neq 1$ for all 
$k \in \mathbb{Z}_{+}$. Since $w_{k} \in\left(1-t_{k}\right) T\left(u_{k}\right)$ we have $\left\langle w_{k}-\left(1-t_{k}\right) y, u_{k}-x\right\rangle \geq 0$ for all $(x, y) \in \mathcal{G}(T)$ and for all $k \in \mathbb{Z}_{+}$. As $k \rightarrow \infty$ we get the inequality

$$
\liminf \left\langle w_{k}, u_{k}-x\right\rangle \geq 0
$$

for all $x \in D(T)$. Consequently

$$
\langle w, u\rangle \geq \lim \sup \left\langle w_{k}, u_{k}\right\rangle \geq \liminf \left\langle w_{k}, u_{k}\right\rangle \geq\langle w, x\rangle
$$

for all $x \in D(T)$. Since $D(T)$ is dense in $X$, we finally obtain

$$
\langle w, u-x\rangle \geq 0 \text { for all } x \in X .
$$

From this we conclude that $w=0 \in T_{1}(u)$. Moreover, by the above calculations with $w=0$ we get $0=\langle w, x\rangle \geq \liminf \left\langle w_{k}, u_{k}\right\rangle$ and thus $\lim \left\langle w_{k}, u_{k}\right\rangle=0$, i.e., $\lim \left\langle w_{k}, u_{k}-u\right\rangle=0$, completing the proof.

\section{UNIQUENESS OF DEGREE}

Let $\hat{d}$ be another degree function defined for the same class of mappings as $d$ satisfying the properties (a)-(d). Let $T: D(T) \rightarrow 2^{X^{*}} \backslash \emptyset$ with $0 \in T(0)$ be a maximal monotone multivalued map and $F: \bar{G} \rightarrow X^{*}$ a bounded demicontinuous map which is of class $\left(S_{+}\right)$, where $G$ is an open bounded set in $X$. Assume that $y_{0} \notin(T+F)(\partial G \cap D(T))$. We have to show that

$$
\mathrm{d}\left(T+F, G, y_{0}\right)=\hat{\mathrm{d}}\left(T+F, G, y_{0}\right) .
$$

By definition we can write

$$
\mathrm{d}\left(T+F, G, y_{0}\right)=\mathrm{d}_{S_{+}}\left(T_{\tilde{\varepsilon}}+F, G, y_{0}\right)
$$

for some fixed $\tilde{\varepsilon}>0$, which is chosen sufficiently small. The restriction of d (or $\hat{\mathrm{d}}$ ) to bounded demicontinuous maps of class $\left(S_{+}\right)$(corresponding the case $T=0$ ) defines a topological degree $\mathrm{d}_{S_{+}}$which is unique [1], 4]. Hence $\mathrm{d}$ and $\hat{\mathrm{d}}$ coincide on that smaller class and especially

$$
\mathrm{d}\left(T_{\tilde{\varepsilon}}+F, G, y_{0}\right)=\mathrm{d}_{S_{+}}\left(T_{\tilde{\varepsilon}}+F, G, y_{0}\right)=\hat{\mathrm{d}}\left(T_{\tilde{\varepsilon}}+F, G, y_{0}\right) .
$$

For the next, crucial step, we need the following result. Note that we do not impose any condition on the domain $D(T)$.

Lemma 6.1. Let $T: D(T) \rightarrow 2^{X^{*}} \backslash \emptyset, 0 \in T(0)$, be a maximal monotone map and $\tilde{\varepsilon}>0$ fixed. Define

$$
H_{t}=\left(T^{-1}+t \tilde{\varepsilon} J^{-1}\right)^{-1}, \quad 0 \leq t \leq 1,
$$

where $D\left(H_{t}\right)=X$ for $0<t \leq 1$ and $D\left(H_{0}\right)=D(T)$. Then $H_{t}, 0 \leq t \leq 1$, defines a pseudomonotone homotopy of maximal monotone mappings.

Proof. First note that $H_{t}$ is maximal monotone for all $0 \leq t \leq 1$ and $H_{t}$ is single valued for all $0<t \leq 1$. We use condition (3) of Lemma4.1 to prove the proposition. Let $\left(t_{k}\right) \subset[0,1], t_{k} \rightarrow t \neq 0$ and $w=H_{t}(u)$. We set $u_{k} \equiv u$ and $w_{k}=H_{t_{k}}\left(u_{k}\right)=$ $H_{t_{k}}(u)$. Then $u_{k} \rightarrow u$ and by Lemma 3.2 also $w_{k} \rightarrow w$. Next let $\left(t_{k}\right) \subset[0,1]$, $t_{k} \rightarrow 0$ and $(u, w) \in \mathcal{G}\left(H_{0}\right)$, that is, $u \in D(T)$ and $w \in T(u)$. Without loss of generality we can assume that $t_{k} \neq 0$ for all $k \in \mathbb{Z}_{+}$. This time we set $w_{k} \equiv w$ and $u_{k}=u+t_{k} \tilde{\varepsilon} J^{-1}(w)$. Now $w_{k} \in T\left(u_{k}-t_{k} \tilde{\varepsilon} J^{-1}\left(w_{k}\right)\right)$ which is equal to $w_{k}=w=$ $H_{t_{k}}\left(u_{k}\right)$. Since $u_{k} \rightarrow u$ and $w_{k} \rightarrow w$ the proof is completed. 
Since $y_{0} \notin\left(H_{t}+F\right)\left(\partial G \cap D\left(T_{t}\right)\right)$ for all $0 \leq t \leq 1$, we obtain by the homotopy invariance of $\hat{\mathrm{d}}$ that

$$
\hat{\mathrm{d}}\left(H_{1}+F, G, y_{0}\right)=\hat{\mathrm{d}}\left(H_{0}+F, G, y_{0}\right),
$$

that is,

$$
\hat{\mathrm{d}}\left(T_{\tilde{\varepsilon}}+F, G, y_{0}\right)=\hat{\mathrm{d}}\left(T+F, G, y_{0}\right) .
$$

Hence we have proved the desired equality

$$
\mathrm{d}\left(T+F, G, y_{0}\right)=\mathrm{d}\left(T_{\tilde{\varepsilon}}+F, G, y_{0}\right)=\hat{\mathrm{d}}\left(T_{\tilde{\varepsilon}}+F, G, y_{0}\right)=\hat{\mathrm{d}}\left(T+F, G, y_{0}\right) .
$$

\section{REFERENCES}

[1] J. Berkovits, On the degree theory for mappings of monotone type, Ann. Acad. Sci. Fenn. Ser. A I Math. Dissertationes, 58 (1986). MR846256 (87f:47084)

[2] J. Berkovits and V. Mustonen, Nonlinear Mappings of Monotone Type, I. Classification and Degree Theory, Report No. 2/88, Mathematics, University of Oulu (1988), 53 pp.

[3] J.M. Borwein, Maximality of sums of two maximal monotone operators, Proc. Amer. Math. Soc., 134(10) (2006), 2951-2955. MR2231619

[4] F.E. Browder, Fixed point theory and nonlinear problems, Proc. Sympos. Pure Math. (39) Part 2, AMS, Providence, RI, 1983, pp. 49-87.

[5] K. Deimling, Nonlinear Functional Analysis, Springer-Verlag, Berlin, 1985. MR787404 (86j:47001)

[6] S. Hu and N. Papageorgiou, Generalizations of Browder's degree theory, Trans. Amer. Math. Soc., 347(1) (1995), 233-259. MR1284911 (96e:47068)

[7] M. Otani and J. Kobayashi, Topological degree for $(S)_{+}-$mappings with maximal monotone perturbations and its applications to variational inequalities, Nonlinear Anal., 59 (2004), 147172. MR2092083 (2005f:49028)

[8] E. Zeidler, Nonlinear functional analysis and its applications. II/B, in "Nonlinear Monotone Operators", Springer-Verlag, New York, 1990. MR1033498(91b:47002)

Department of Mathematical Sciences, University of Oulu, P.O. Box 3000, FiN-90014 Oulu, Finland

Department of Mathematical Sciences, University of Oulu, P.O. Box 3000, Fin-90014 Oulu, Finland

E-mail address: circus@mail.student.oulu.fi 